\title{
Early potential effects of resveratrol supplementation on skeletal muscle adaptation involved in exercise-induced weight loss in obese mice
}

\author{
Jingyu Sun,"\#, Chen Zhang ${ }^{2, \#}$, MinJeong Kim², Yajuan Su ${ }^{4}$, Lili Qin ${ }^{1}$, Jingmei Dong ${ }^{1}$, Yunhe Zhou ${ }^{1}$ E Shuzhe Ding ${ }^{5, *}$ \\ ${ }^{1}$ Sports and Health Research Center, Tongji University Department of Physical Education, Shanghai 200092, ${ }^{2}$ Tongji University School of \\ Medicine, Shanghai 200092, China, ${ }^{3}$ Chung-Ang University College of Medicine, Seoul 06973, Korea, ${ }^{4}$ Tongji University School of Life \\ Sciences and Technology, Shanghai 200092, ${ }^{5}$ Key Laboratory of Adolescent Health Assessment and Exercise Intervention, East China \\ Normal University, Shanghai 200241, China
}

\begin{abstract}
Exercise and resveratrol supplementation exhibit anti-obesity functions in the long term but have not been fully investigated yet in terms of their early potential effectiveness. Mice fed with high-fat diet were categorized into control (Cont), exercise (Ex), resveratrol supplementation (Res), and exercise combined with resveratrol supplementation (Ex + Res) groups. In the four-week period of weight loss, exercise combined with resveratrol supplementation exerted no additional effects on body weight loss but significantly improved whole-body glucose and lipid homeostasis. The combined treatment significantly decreased intrahepatic lipid content but did not affect intramyocellular lipid content. Moreover, the treatment significantly increased the contents of mtDNA and cytochrome $c$, the expression levels of peroxisome proliferator-activated receptor gamma coactivator-1 alpha and its downstream transcription factors, and the activities of ATPase and citrate synthase. However, exercise, resveratrol, and their combination did not promote myofiber specification toward slow-twitch type. The effects of exercise combined with resveratrol supplementation on weight loss could be partly due to enhanced mitochondrial biogenesis and not to fiber-type shift in skeletal muscle tissues. [BMB Reports 2018; 51(4): 200-205]
\end{abstract}

\section{INTRODUCTION}

The prevalence of overweight and obesity has increased

*Corresponding author. Tel: +86-21-2059-5005; Fax: +86-21-20595019; E-mail: szding@tyxx.ecnu.edu.cn

${ }^{\#}$ These authors contributed equally to this work.

https://doi.org/10.5483/BMBRep.2018.51.4.236

Received 27 December 2017, Revised 18 January 2018, Accepted 2 March 2018

Keywords: Endurance exercise, Glucose and lipids homeostasis, Resveratrol, Skeletal muscle adaptation, Weight loss considerably in many countries over the past three decades. Obesity and its associated metabolic disorders are a major public health concern. Therefore, effective preventive and therapeutic strategies must be developed to counteract obesity and its adverse consequences. Exercise (1) and calorie restriction (2) can attenuate the extent of obesity. Similar to exercise training and calorie restriction, resveratrol (3-5-4'-trihydroxy-trans-stilbene) treatment is widely accepted because it affects energy metabolism (3). Moreover, resveratrol is an important antioxidant that can eliminate reactive oxygen species accumulation induced by a high-fat diet (HFD) (4). Thus, speculations indicate that resveratrol supplementation might augment the effects of exercise on weight loss in the long term; however, the early potential efficacy of the combined treatment has not been fully understood yet.

In addition to designing interventions to improve obese status, identifying the underlying molecular mechanisms is equally important. HFD induces obesity partially by decreasing the mitochondrial number and impairing the oxidative capacity in skeletal muscles (5). Given that the oxidative capacity of skeletal muscles is predominately dependent on mitochondria, increasing lines of evidence suggest that mitochondrial biogenesis and the associated fatty acid oxidation enhancement may directly improve whole-body glucose and lipid hemostasis (6). This effect has been observed in patients with insulin resistance and type 2 diabetes $(7,8)$ and in several animal models (9). Hence, promoting mitochondrial biogenesis via specific approaches, such as endurance training combined with resveratrol supplementation, could be a promising therapeutic strategy against HFD-induced obesity. Peroxisome proliferator-activated receptor gamma coactivator-1 alpha (PGC-1 $\alpha$ ) is an essential regulator involved in mitochondrial biogenesis (10). In this regard, the present work investigated PGC- $1 \alpha$ and its downstream transcription factors to explore the mechanism of combined treatmentinduced skeletal muscle adaptions in the early phase of weight loss. Mitochondrial oxidative enzymes and muscular phenotypic changes were also assessed. 


\section{RESULTS}

Resveratrol supplementation combined with exercise improves whole-body glucose and lipid homeostasis in the early phase of weight loss but did not augment the individual effects on final body weight loss

Daily cumulative food consumption was not significantly different (Fig. 1B) but body weight loss significantly varied (Fig. $1 \mathrm{~A})$ among the control (Cont), exercise (Ex), resveratrol supplementation (Res), and exercise combined with resveratrol supplementation ( $\mathrm{Ex}+\mathrm{Res}$ ) groups. Interestingly, endurance exercise or resveratrol supplementation alone significantly influenced the final body weight loss, thereby suggesting that resveratrol supplementation produced exercise-like effects on weight loss. However, the combination of exercise training and resveratrol supplementation showed no further effect on the final body weight loss possibly because 4 weeks of resveratrol treatment was insufficient to induce additional effects on the final body weight loss. Interestingly, the weight of epididymal fat significantly decreased (Fig. 1C). Glucose tolerance during the glucose tolerance test (GTT) was significantly improved, as indicated by a reduction in the total area under the curve (AUC) in the Ex + Res group compared with the control group (Fig. 1D). This outcome suggests that endurance exercise combined with resveratrol supplementation

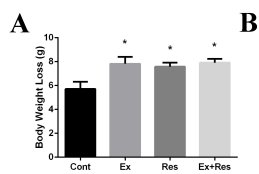

D

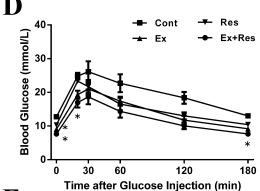

F

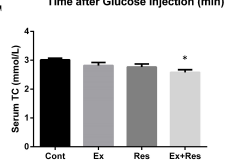

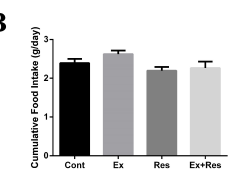

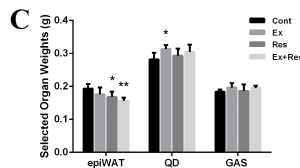

$\mathbf{E}$

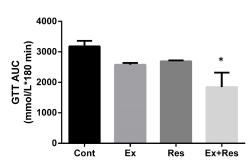

G

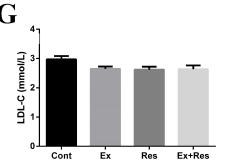

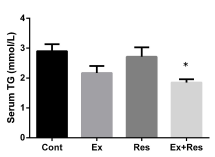

$\mathbf{H}$

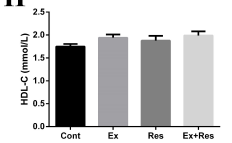

Fig. 1. Exercise combined with resveratrol supplementation improved whole-body glucose and lipid homeostasis in obese mice undergoing weight loss. Body weight loss (A) and daily food intake (B) were recorded during the body weight loss program. The selected tissue weights $(C)$, intraperitoneal glucose tolerance test $(\mathrm{GTT})(\mathrm{D})$, and levels of serum TG (E), TC (F), LDL-C (G), and HDL-C (H) were measured by enzymatic colorimetric methods after the four-week body weight loss program. The weight loss programs of the HFD-fed mice were performed as follows: control (Cont), exercise (Ex), resveratrol supplementation (Res), and exercise combined with resveratrol supplementation (Ex + Res). AUC indicates the total area under the GTT curve. The values represent mean \pm SEM $(n=8)$. ${ }^{*} \mathrm{P}<$ 0.05 , and $* * P<0.01$ vs. Cont group. Epididymal fat (epiWAT), quadriceps femoris muscle (QD), gastrocnemius muscle (GAS). could protect mice from diet-induced glucose intolerance. Furthermore, the levels of serum triglycerides (TG) and total cholesterol (TC) in HFD-fed mice were significantly reduced by the combined endurance exercise and resveratrol supplementation compared with those in the control mice (Figs. 1E and F); however, no changes were observed in the low-density lipoprotein (LDL-C) and high-density lipoprotein (HDL-C) levels (Figs. 1G and H). These data demonstrate that a four-week combined treatment of exercise and resveratrol supplementation is sufficient to improve HFD-induced lipid disturbance and glucose tolerance.

Exercise combined with resveratrol supplementation relieves lipid accumulation in the liver but not in the skeletal muscle of obese mice undergoing weight loss

Furthermore, the abnormal lipid accumulation in non-adipose tissues, particularly liver and skeletal muscles, was evaluated in the current study. The combination of exercise and resveratrol significantly reduced the intrahepatic lipid (IHL) content of HFD-fed mice subjected to the four-week body weight loss program, as evident in the low liver TG values (Fig.

A
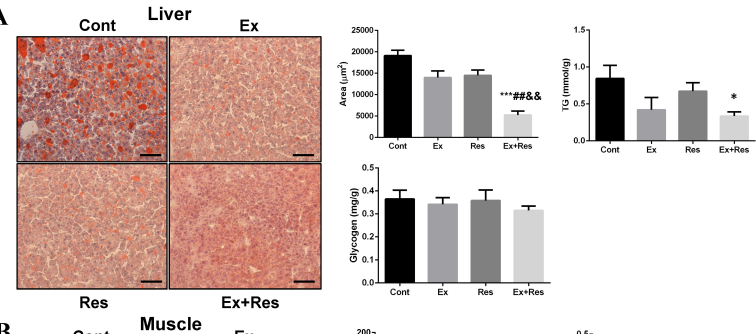

B
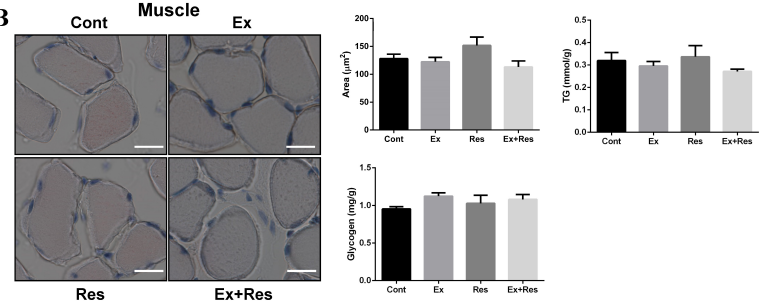

Fig. 2. Exercise combined with resveratrol supplementation relieved lipid accumulations in liver but not in skeletal muscle in obese mice undergoing weight loss. Lipid accumulations in liver (A) and skeletal muscle (B) were detected by Oil Red $\mathrm{O}$ staining, along with the calculation of lipid droplet areas $\left(\mu \mathrm{m}^{2}\right)$ with the Image J system and TG content. Glycogen content was also determined in HFD-fed mice after the four-week body weight loss programs: control (Cont), exercise (Ex), resveratrol supplementation (Res), and exercise combined with resveratrol supplementation (Ex + Res). The representative images of Oil Red $\mathrm{O}$ staining are shown with $20 \times$ magnification, and the scale bars represent $50 \mu \mathrm{m}$ in liver. The representative images of Oil Red $\mathrm{O}$ staining are shown with $100 \times$ magnification, and the scale bars represent $200 \mu \mathrm{m}$ in skeletal muscle. The values represent mean \pm SEM $(n=6)$. P values were calculated by one-way ANOVA. ${ }^{*} \mathrm{P}<0.05$, and $* * * P<0.001$ vs. Cont mice; ${ }^{\# \# P}<0.01$ vs. Ex mice; ${ }^{\&}{ }^{\prime} P<$ 0.01 vs. Res mice. 
2A). These results indicated an improvement in liver lipid homeostasis. Unexpectedly, the contents of IMCL and TG did not significantly decrease in the skeletal muscles of obese mice undergoing weight loss (Fig. 2B). A likely explanation is that a large amount of fat is continuously liberated from peripheral depots to the skeletal muscle for oxidation in the early phase of the combined and single treatment. As a result, the mobilization of IMTG is limited and leads to unchanged IMCL content temporarily. Furthermore, the glycogen content did not significantly change. These findings could be due to insufficiency of the four-week separate or combined treatment in producing sharp responses in the skeletal muscles. Overall, these findings indicate that endurance exercise combined with resveratrol supplementation relieves lipid accumulation in the liver but not in the skeletal muscles of obese mice undergoing weight loss.

\section{Exercise combined with resveratrol supplementation improves skeletal muscle mitochondrial biogenesis in obese mice undergoing weight loss}

To investigate the relationship between muscle metabolic adaptation and mitochondrial quantity, we quantified mtDNA via RT-PCR analysis. The mtDNA content (normalized by nDNA) was upregulated by the combined exercise and resveratrol supplementation (Fig. 3A). Considering that PGC $-1 \alpha$ is the master regulator of mitochondrial biogenesis (10), we further investigated the effect of exercise combined with resveratrol supplementation on PGC- $1 \alpha$ and its downstream
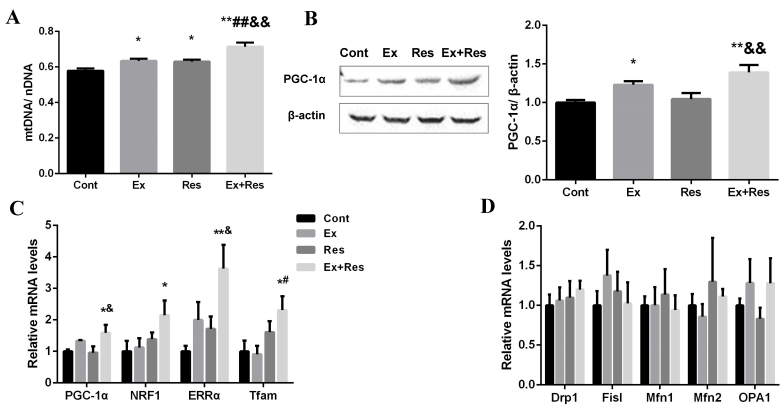

Fig. 3. Exercise combined with resveratrol supplementation increased muscle mitochondrial biogenesis in obese mice undergoing weight loss. Mitochondrial DNA content in skeletal muscle was detected by RT-PCR (A). Western blot and quantitative analyses for PGC- $1 \alpha$ were normalized by $\beta$-actin (B). Relative mRNA expression levels of PGC- $1 \alpha$ and its downstream transcription factors in skeletal muscle were analyzed by RT-PCR (C). Relative mRNA levels of genes related to mitochondrial dynamics were analyzed by RT-PCR (D) in the skeletal muscle tissues of HFD-fed mice in the following four-week body weight loss-programs: control (Cont), exercise (Ex), resveratrol supplementation (Res), and exercise combined with resveratrol supplementation $($ Ex + Res). The values represent mean \pm SEM $(n=6)$. P values were calculated by one-way ANOVA. $* \mathrm{P}<0.05$, and $* * \mathrm{P}<$ 0.01 vs. Cont mice; ${ }^{\#} \mathrm{P}<0.05$, and ${ }^{\# \#} \mathrm{P}<0.01$ vs. Ex mice; ${ }^{\circledR} \mathrm{P}<$ 0.05 , and \&\& $P<0.01$ vs. Res mice. transcription factors in skeletal muscles. As predicted, the mRNA and protein expression levels of PGC-1 $\alpha$ were more upregulated in the Ex + Res group than in the Cont group (Fig. 3B). The downstream transcription factors of PGC- $1 \alpha$, including nuclear respiratory factor 1 (NRF-1), estrogen-related receptor $\alpha(E R R \alpha)$, and mitochondrial transcription factor $A$ (Tfam), were also upregulated (Fig. 3C). The transcript levels of dynamin-related protein-1 (Drp1), fission-1 (Fis1) related to mitochondrial fission, and mitofusins (Mfn1, Mfn2) and optical atrophy-1 (OPA1) related to mitochondrial fusion were not changed (Fig. 3D). These results showed that the combined treatment might promote mitochondrial biogenesis but not mitochondrial dynamics in the process of weight loss.

\section{Exercise combined with resveratrol supplementation increases mitochondrial oxidative enzyme activity and content in the skeletal muscles of obese mice undergoing weight loss}

To preliminarily explore the mitochondrial oxidative capacity during the four-week body weight loss programs, we determined the protein levels of carnitine palmitoyl transferase- 1 (CPT-1, CPT-1M as a prominent skeletal muscle-specific isoform) and pyruvate dehydrogenase kinase 4 (PDK4) by Western blot
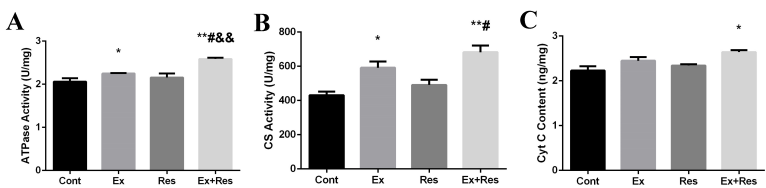

D

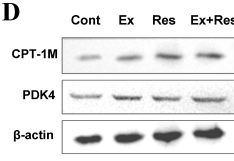

$\mathbf{E}$

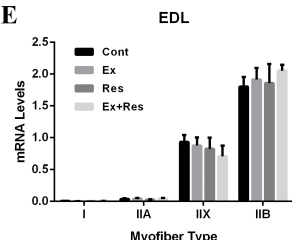

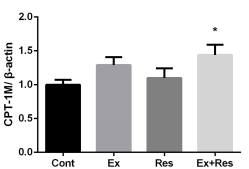

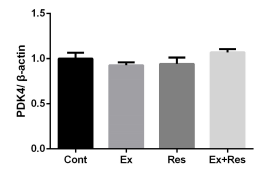

SOL

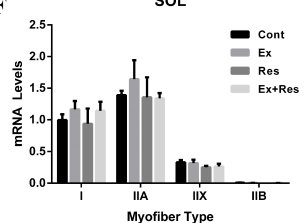

Fig. 4. Exercise combined with resveratrol supplementation upregulated mitochondrial oxidative enzymes but did not promote myofiber specification toward slow-twitch type. The activity of ATPase (A) and CS (B) were examined by microplate method. Cty $\mathrm{C}$ content (C) was determined by ELISA. Western blot and quantitative analyses for CPT-1M and PDK4 were normalized by $\beta$-actin (D). MyHC isoforms with mRNA normalized against $\beta$-actin in the fast-twitch extensor digitorum longus (EDL) (E) and slow-twitch soleus (SOL) (F) muscles were detected after the four-week body weight loss programs: control (Cont), exercise $(E x)$, resveratrol supplementation (Res), and exercise combined with resveratrol supplementation (Ex + Res). The bars represent mean \pm SEM $(n=6)$. $P$ values were calculated by one-way ANOVA. ${ }^{* P}<0.05$, and ${ }^{* * P}<0.01$ vs. Cont mice; ${ }^{\# P}<$ 0.05 vs. Ex mice; ${ }^{\& \&} \mathrm{P}<0.01$ vs. Res mice. 
analysis. The combination of exercise and resveratrol significantly increased the protein expression levels of CPT-1M but did not affect the protein expression levels of PDK4 relative to those in the control mice (Fig. 4D). This finding could be due to the fact that FA might be the predominant substrate for muscle mitochondrial oxidation utilization during the weight loss process in the combined treatment. In addition to CPT-1M, the activities of ATPase and citrate synthase (CS) and the content of cytochrome c (Cyt C) evidently increased in the skeletal muscles of Ex + Res mice than in the control mice (Figs. 4A, B, and C). Furthermore, mice subjected to exercise alone showed significantly increased ATPase and CS activities compared with the control mice. These findings indirectly indicated that single and combined treatment improved the capacity of mitochondrial oxidation, which might partly contribute to the early weight loss process of obese mice.

\section{Endurance exercise, resveratrol, or combined treatment regimens does not promote myofiber specification toward slow-twitch type in obese mice undergoing weight loss} To further explore the effects of exercise combined with resveratrol supplementation on changes in muscle fiber type of obese mice undergoing weight loss, we determined the expression pattern of $\mathrm{MyHC}$ isoform in fast- and slow-switch skeletal muscles through RT-PCR analysis. The fast-twitch extensor digitorum longus (EDL) muscles consisted of the four isoforms but were dominated by IIx (Myh1) and Ilb (Myh4) fibers; meanwhile, slow-twitch soleus (SOL) muscles were dominated by type I (Myh7) and Ila (Myh2) fibers (Figs. 4E and F). Furthermore, endurance exercise, resveratrol, or their combination neither suppressed the expression of fast $\mathrm{MyHC}$ transcript nor enhanced the expression of $\mathrm{MyHC}$ slow transcript in EDL and SOL muscles. Hence, these treatments did not promote myofiber specification toward slow-twitch type.

\section{DISCUSSION}

Metabolic abnormalities may be caused by abnormal fat accumulation in non-adipose tissues, particularly liver and skeletal muscles (11). Recent study demonstrates that endurance exercise (12) and resveratrol supplementation (13) can decrease IHL content. In the present work, the IHL content significantly decreased in HFD-fed mice after the four-week combined treatment of exercise and resveratrol supplementation. In addition to other beneficial metabolic adaptations, such as lowering of circulating and liver TG content and improving glucose tolerance, the present data hinted that the combined treatment improved whole-body glucose and lipid homeostasis. Except for the IHL content, an inverse relationship between IMCL and insulin sensitivity has been reported (14). Interestingly, the contents of IMCL and TG did not decrease in HFD-fed mice after the four-week combined or separate treatment. These findings are consistent with previous observations (15). The absence of any reduction in the IMTG content by single or combined treatment may represent a positive adaptation mechanism, which contributes to lipid utilization during the early phase of weight loss (16). We speculated the pronounced reduction in the IMTG content in the middle and later phase of the treatment, and this finding will be further studied in the future.

To explain the early potential effects of the combined treatment on muscle metabolic adaptation, we measured key regulators related to mitochondrial quantity and function. The impaired ability of skeletal muscles to oxidize FA with obesity could be associated with reduced mitochondrial content or impaired mitochondrial biogenesis $(17,18)$. Thus, the increased expression of general transcription factors that regulate mitochondrial content and mitochondrial biogenesis could be responsible for the effectiveness of anti-obesity treatment. Here, exercise, resveratrol, or their combination upregulated the mitochondrial number (as demonstrated by the mtDNA copy number). Significant upregulation was detected when administering the combined treatment. Hence, the combined treatment might be a potential therapeutic strategy to relieve obesity. Moreover, the coordination between mtDNA and nuclear DNA transcription is a key component of mitochondrial biogenesis. PGC- $1 \alpha$ is a pivotal regulator of mitochondrial biogenesis (19). Exercise (20) and in vitro electrical stimulation (21) promote mitochondrial biogenesis primarily by upregulating PGC- $1 \alpha$ expression. Similarly, resveratrol intake stimulates mitochondrial biogenesis by increasing PGC- $1 \alpha$ expression (22). In the present study, exercise combined with resveratrol supplementation increased muscle PGC- $1 \alpha$ expression at both transcript and protein levels. In addition to PGC- $1 \alpha$, its downstream transcription factors, including NRF-1, ERR, and Tfam, were upregulated, indicating that the combined treatment promoted mitochondrial biogenesis in the early period of weight loss. However, we did not observe changes in the transcript levels of genes related to mitochondrial fusion and fission. Overall, data suggest that increased mitochondrial content, but not those caused by intrinsic mitochondrial alterations, is associated with the early process of weight loss by the combined treatment. This distinction is important because it suggests that combined interventions are required to enhance overall mitochondrial biogenesis without any "remodeling" of existing mitochondria and promote weight loss.

CPT-1 is a rate-limiting step in mitochondrial FA uptake. The combined treatment of endurance exercise and resveratrol supplementation significantly increased the CPT-1M protein expression. This finding provides an indirect support to the notion that the combined treatment helps augments anti-obesity benefits by promoting FA to transport into muscle mitochondria. Additionally, the content and activity of mitochondrial oxidative enzymes are critical indicators of FA oxidation. As predicted, exercise combined with resveratrol supplementation significantly increased muscle ATPase 
activity, CS activity, and Cyt C content in the process of weight loss; such changes are consistent with the alterations in mtDNA content and PGC- $1 \alpha$ expression. Reports show that interventions for amplifying muscle PGC- $1 \alpha$ expression can stimulate a shift from fast glycolytic to slow oxidative phenotype in obese mice (23). The increase in the PGC- $1 \alpha$ expression induced by the combined treatment was associated with lack of changes in myofiber specification shift. Two possibilities exist. First and most likely, the treatment dose and duration were insufficient to evoke robust muscle remodeling, although the PGC- $1 \alpha$ protein expression increased. Second, the body's response to exercise stimulation is systemic and multidimensional. Except for PGC- $1 \alpha$, other regulatory molecules produced by the combined treatment might contribute to the blocking of myofiber specification toward slow-twitch type.

In conclusion, endurance exercise combined with resveratrol supplementation improved whole-body glucose and lipid homeostasis and increased the expression levels of PGC- $1 \alpha$ and its downstream transcription factors. Such change is likely to induce some metabolic adaptations in the skeletal muscles, including an increase in mitochondrial quantity and mitochondrial oxidative enzymes by improving mitochondrial biogenesis. However, no change in myofiber specification shift was observed. Aside from muscle mitochondrial biogenesis, other mechanisms involved in the beneficial effects of exercise combined with resveratrol supplementation on weight loss are worth studying in the future.

\section{MATERIALS AND METHODS}

\section{Animal care and manipulation}

ICR/JCL male mice ( $n=32,8$ weeks old) were purchased from SLAC Laboratory Animal Research Center (Shanghai, China) and housed in a controlled environment $(12 / 12 \mathrm{~h}$ light/dark cycle, humidity: $60 \% \pm 5 \%$, temperature: $23 \pm 2^{\circ} \mathrm{C}$ ). All mice were provided with free access to saturated HFD for six weeks to gain weight and were then randomly divided into four groups to lose weight: (1) Cont $(n=8)$, (2) $\operatorname{Ex}(n=8)$, (3) Res $(n=8)$, and (4) Ex $+\operatorname{Res}(n=8)$. Exercise training was conducted using a motor treadmill at a moderate intensity (1.3 $\mathrm{km} / \mathrm{h}$ ) for six days a week for four weeks at $60 \mathrm{~min} /$ day. Commercially dried resveratrol (Sigma, MO, USA) was used and dissolved in $0.5 \%$ carboxymethyl cellulose (CMC) solution. The oral gavage with resveratrol $(25 \mathrm{mg} / \mathrm{kg}$ body weight) was performed in the Res and Ex + Res groups $1 \mathrm{~h}$ after exercise. The Cont and Ex groups were orally administered with $0.5 \% \mathrm{CMC}$ as vehicle. After the four-week body weight loss programs, intraperitoneal GTT was performed after a 16-hour fasting by injection of glucose (1 $\mathrm{g} / \mathrm{kg}$ ). The animal study was supervised by the Animal Care and Use Ethics Committee of East China Normal University.

\section{Lipids and glycogen content measurement}

At the end of their respective manipulation, all mice were fasted overnight. Blood samples were collected, and serum TG, TC, LDL-C, and HDL-C levels were analyzed using corresponding commercial kits (Shanghai Rongsheng Biotech Co., Shanghai, China) as previously described (24). For the analysis of glycogen and TG content in liver and muscle, the supernatant of homogenate was removed for measurement according to the kits' protocol (Shanghai Rongsheng Biotech Co., Shanghai, China).

\section{Oil Red O staining}

The liver and muscle samples were fixed in $4 \%$ paraformaldehyde for $1 \mathrm{~h}$ at $4^{\circ} \mathrm{C}$. Samples were incubated in $30 \%$ sucrose solution overnight and then embedded with O.C.T. compound. After blotting and staining with filtered Oil Red O solution, slides were mounted and covered with glycerol gelatin.

\section{Determination of mitochondrial oxidative enzymes}

The activities of ATPase and CS and Cyt C content were assayed using corresponding assay kits (Abcam, MA, USA) following the manufacturer's instructions. Detergent (1/10 vol) was added to the mitochondria to extract transmembrane proteins. Absorbance was recorded with a TECAN microplate reader (Molecular Device, Switzerland).

\section{RNA extraction and semi-quantitative RT-PCR}

Total RNA was isolated and reverse transcribed as previously described (24). Primer pairs were designed on the basis of GenBank reference sequences and listed in the Supplementary Material (Table S1). The mRNA abundance of the targeted genes was normalized to that of $\beta$-actin.

\section{mtDNA quantification}

mtDNA and total DNA were extracted and purified from tissues by the method described previously (25). ND1 was used as mtDNA marker, and Pecam1 was used as a nuclear DNA marker. Primer pairs were designed on the basis of GenBank reference sequences and listed in the Supplementary Material (Table S1).

\section{Westem blot analysis}

The protein content determination and Western blot analysis were performed as previously described (24). The following primary antibodies were used: anti-PGC-1 $\alpha$ (H-300) (sc-13067), anti-CPT-1M (H-120) (sc-20670), and anti-PDK4 (N-15) (sc-14492) (Santa Cruz Biotechnology, CA, USA). The protein bands were visualized with a chemiluminescence reagent (ECL, Cell Signaling, Beverly, MA, USA) and quantified using Image J software.

\section{Statistical analysis}

Data were expressed as mean \pm SEM. The differences in 
means were analyzed by one-way ANOVA. The level of significance was set at $P<0.05$. All data analyses were performed using the SPSS 20.0 statistical package (Chicago, IL, USA).

\section{ACKNOWLEDGEMENTS}

This study was supported by the National Natural Science Foundation of China (Nos. 31600966, 31671241, 31401019, and 31771313), the scientific fitness guidance project of the National Sports General Administration of China (2017B026), and the Tengfei project of the Shanghai Municipal Sports Bureau (16T006).

\section{CONFLICTS OF INTEREST}

The authors have no conflicting interests.

\section{REFERENCES}

1. Chin SH, Kahathuduwa CN and Binks M (2016) Physical activity and obesity: what we know and what we need to know. Obes Rev 17, 1226-1244

2. Normandin E, Chmelo E, Lyles MF, Marsh AP and Nicklas BJ (2017) Effect of Resistance Training and Caloric Restriction on the Metabolic Syndrome. Med Sci Sports Exerc 49, 413-419

3. Timmers S, Konings E, Bilet $\mathrm{L}$ et al (2011) Calorie restriction-like effects of 30 days of resveratrol supplementation on energy metabolism and metabolic profile in obese humans. Cell Metab 14, 612-622

4. Haohao Z, Guijun Q, Juan Z, Wen K and Lulu C (2015) Resveratrol improves high-fat diet induced insulin resistance by rebalancing subsarcolemmal mitochondrial oxidation and antioxidantion. J Physiol Biochem 71, 121-131

5. Sparks LM, Xie H, Koza RA et al (2005) A high-fat diet coordinately downregulates genes required for mitochondrial oxidative phosphorylation in skeletal muscle. Diabetes 54, 1926-1933

6. Civitarese AE, Ukropcova B, Carling S et al (2006) Role of adiponectin in human skeletal muscle biogenesis. Cell Metab 4, 75-87

7. Mootha VK, Lindfren CM, Eriksson KF et al (2003) PGC-1alpha-responsive genes involved in oxidative phosphorylation are coordinately downregulated in human diabetes. Nat Genet 34, 267-273

8. Heilbronn LK, Gan SK, Turner N, Campbell LV and Chisholm DJ (2007) Markers of mitochondrial biogenesis and metabolism are lower in overweight and obese insulin-resistant subjects. J Clin Endocrinol Metab 92, 1467-1473

9. Bonnard C, Durand A, Peyrol S et al (2008) Mitochondrial dysfunction results from oxidative stress in skeletal muscle of diet-induced insulin resistant mice. J Clin Invest 118, 789-800
10. Benton CR, Wright DC and Bonen A (2008) PGC-1alphamediated regulation of gene expression and metabolism: implications for nutrition and exercise prescriptions. Appl Physiol Nutr Metab 33, 843-862

11. Despres JP, Lemieux I, Bergeron J et al (2008) Abdominal obesity and the metabolic syndrome: contribution to global cardiometabolic risk. Arterioscler Thromb Vasc Biol 28, 1039-1049

12. Brouwers $B$, Hesselink MK, Schrauwen $P$ and SchrauwenHinderling VB (2016) Effects of exercise training on intrahepatic lipid content in humans. Diabetologia 59, 2068-2079

13. Ahn J, Cho I, Kim S, Kwon D and Ha T (2008) Dietary resveratrol alters lipid metabolism-related gene expression of mice on an atherogenic diet. J Hepatol 9, 1019-1028

14. Savage DB, Petersen KF and Shulman Gl (2007) Disordered lipid metabolism and the pathogenesis of insulin resistance. Physiol Rev 87, 507-520

15. Helge JW and Dela F (2003) Effect of training on muscle triacylglycerol and structural lipids: a relation to insulin sensitivity? Diabetes 52, 1881-1887

16. Goodpaster BH, He J, Watkins S and Kelley DE (2001) Skeletal muscle lipid content and insulin resistance: evidence for a paradox in endurance-trained athletes. J Clin Endocrinol Metab 86, 5755-5761

17. Boushel R, Gnaiger E, Schjerling P, Skovbro M, Kraunsoe $R$ and Dela F (2007) Patients with type 2 diabetes have normal mitochondrial function in skeletal muscle. Diabetologia 50, 790-796

18. Holloway GP, Thrush AB, Heigenhauser GJ et al (2007) Skeletal muscle mitochondrial FAT/CD36 content and palmitate oxidation are not decreased in obese women. Am J Physiol Endocrinol Metab 292, E1782-1789

19. $\mathrm{Wu} Z$, Puigserver $P$, Andersson $U$ et al (1999) Mechanisms controlling mitochondrial biogenesis and respiration through the thermogenic coactivator PGC-1. Cell 98, 115-124

20. Ji LL and Kang C (2015) Role of PGC-1 $\alpha$ in sarcopenia: etiology and potential intervention-a mini-review. Gerontology 61, 139-148

21. Atherton PJ, Babraj J, Smith K, Singh J, Rennie MJ and Wackerhage H (2005) Selective activation of AMPK-PGC1alpha or PKB-TSC2-mTOR signaling can explain specific adaptive responses to endurance or resistance training-like electrical muscle stimulation. FASEB J 19, 786-788

22. Valero T (2014) Mitochondrial biogenesis: pharmacological approaches. Curr Pharm Des 20, 5507-5509

23. Lin J, Wu H, Tarr PT et al (2002) Transcriptional co-activator PGC-1 alpha drives the formation of slow-twitch muscle fibres. Nature 418, 797-801

24. Sun J, Huang T, Qi Z et al (2017) Early Mitochondrial Adaptations in Skeletal Muscle to Obesity and Obesity Resistance Differentially Regulated by High-Fat Diet. Exp Clin Endocrinol Diabetes 125, 538-546

25. O'Neill BT, Kim J, Wende AR et al (2007) A conserved role for phosphatidylinositol 3-kinase but not Akt signaling in mitochondrial adaptations that accompany physiological cardiac hypertrophy. Cell Metab 6, 294-306 